\section{Fabry disease, a complex pathology not easy to diagnose}

Paolo Colomba, ${ }^{1}$ Simone Scalia, ${ }^{1}$

Giuseppe Cammarata, ${ }^{1}$ Carmela Zizzo, ${ }^{1}$

Daniele Francofonte, ${ }^{1}$ Vincenzo Savica,

Riccardo Alessandro, ${ }^{1,2}$

Francesco lemolo, ${ }^{1,3}$ Giovanni Duro'

'Institute of Biomedicine and Molecular

Immunology A. Monroy, Italian National

Research Council, Palermo; ${ }^{2}$ Department

of Medical Biopathology and

Biotechnology, University of Palermo;

${ }^{3}$ Department of Neurology, Guzzardi

Hospital, Vittoria (RG), Italy

\section{Abstract}

Fabry disease is a multisystemic lysosomal storage disorder, inherited in an X-linked manner. It is a defect of metabolism of the glycosphingolipids, due to the reduction or absence of the activity of lysosomal enzyme $\alpha$ galactosidase $\mathrm{A}$. This reduction of activity causes the storage of globotriaosylceramide and derivatives in the lysosomes, triggering a cascade of cellular events, mainly in vascular endothelium. These events are the responsible for the systemic clinical manifestations and the renal, cardiac and cerebrovascular complications, or a combination of them. The symptomatology can lead to the premature death of patient between the fourth or fifth decade of life. The first symptoms can occur at different ages, generally in childhood, with different severity and course. Fabry disease is suspected on the basis of clinical and anamnestic-familial data, and it is confirmed by enzymatic and genetic assays. However, Fabry disease could be a pathology more complex than previously considered, and the diagnostic tests that are currently in use could be not always sufficient to confirm the clinical diagnosis. Probably, other factors could be also involved in the onset of symptomatology. In the last years, the knowledge of the disease is considerably increased but other studies are necessary to make a prompt and reliable diagnosis. An early diagnosis of Fabry disease is essential for the beginning of the enzyme replacement therapy, which can contribute to arrest its progression and improve the quality of life of patients.

\section{Anderson-Fabry: description of the disease}

Anderson-Fabry (or Fabry) disease (FD) takes the name from the two dermatologists that independently described for the first time the pathology at the end of 1800 . It is a lysosomal storage disorder caused by a functional defect of the enzyme $\alpha$-galactosidase A ( $\alpha$-GAL A). ${ }^{1}$ This deficit is responsible for the alteration of the metabolism of some glycosphingolipids, mainly globotriaosylceramide (Gb3) and lyso-Gb3, which consequently build up in the lysosomes of different cellular types, mostly in vascular endothelial cells. ${ }^{2}$ The disease is considered rare and its incidence was estimated at 1:40,000 in general population. However, recent neonatal screening studies showed a higher incidence: about 1 in 3100 among newborn males in Italy ${ }^{3}$ and 1 in 1500 among newborn males in Taiwan. ${ }^{4}$ Fabry disease is caused by mutations in the $G L A$ gene, encoding the $\alpha$ GAL A, located on the long arm of the X-chromosome (Xq21-22). ${ }^{5}$ This localization determines a X-linked transmission of the pathology: this means that an affected male can transmit the defect only to daughters, while an affected woman has $50 \%$ of probability to transmit the $G L A$ gene mutated to her sons and daughters. To date, more than 770 mutations in the GLA coding regions were described in Fabry patients (Human Gene Mutation Database, www.hgmd.org). However, there is not a clear genotype-phenotype correlation.

Generally, the classic clinical manifestations of Fabry disease occur during pediatric age with lancinating acroparesthesias in the extremities. ${ }^{6}$ The autonomic nervous system impairment is responsible for the alteration of sweating, which becomes a critical element in particular situations (like fever or physical exertion): in these moments, patients can manifest the typical crisis of pain, due to the thermoregulation disorder. Also the gastrointestinal pain, associated to diarrhea alternated with sensation of abdominal fullness, is caused by the involvement of the vegetative local system. The skin in Fabry disease can manifest the typical angiokeratomas localized mainly in the swimsuit area or even they can be present as angiokeratomas corporis diffusum. Moreover, patients can have lymphedemas mostly in the lower limbs. The eye, like in many neuro-metabolic diseases, shows different signs such as the typical cornea verticillata, cataract and telangiectasia of the conjunctiva. The storage of glycosphingolipids in renal cells compromise their function, leading to a progressive nephropathy that is one of the most critical manifestation of the disease. ${ }^{7}$ In Fabry patients, the hearth frequently presents a left ventricular hypertrophy, seemingly idiopathic, and a direct impairment of valvular apparatus and intracardiac conduction, with various complications, such as cardiac failure or arrhythmic pathology, which can lead to death caused by myocardial infarction. The endocrine system has been the object of deep investigations: it frequently presents a deficit
Correspondence: Giovanni Duro, Institute of Biomedicine and Molecular Immunology A. Monroy, Italian National Research Council (IBIMCNR), via Ugo La Malfa 153, 90146 Palermo, Italy. E-mail: giovanni.duro@ibim.cnr.it

Key words: Anderson-Fabry disease; $\alpha$-galactosidase A; $G L A$ gene.

Conflict of interest: the authors declare no potential conflict of interest.

Received for publication: 30 October 2015 Revision received: 27 November 2015. Accepted for publication: 29 November 2015.

This work is licensed under a Creative Commons Attribution NonCommercial 3.0 License (CC BYNC 3.0).

(C) Copyright P. Colomba et al., 2015

Licensee PAGEPress, Italy

Cardiogenetics 2015; 5:5612

doi:10.4081/cardiogenetics.2015.5612

related to high vascularity and low cellular turnover that are typical of endocrine tissue. ${ }^{8,9}$ Besides the impairment of the peripheral nervous system, Fabry patients present a direct involvement of the brain with juvenile ischemic strokes and possible hemorrhagic episodes. However, the classic clinical picture, with all the typical manifestations of the disease, is observed only in about $30 \%$ of affected patients. Instead, the $70 \%$ of them is affected by atypical variants of the disease that usually manifest a mild and late-onset phenotype and frequently the involvement of a single organ. Moreover, female patients usually present a milder and more variable symptomatology in comparison to male subjects. In fact, female patients are usually heterozygous and they have organs that are chimeras of normal and affected cells because one of the two X-chromosomes is inactivated (lyonization). For this reason, their symptomatology is milder and therefore these patients are more difficult to diagnose. Until some years ago, affected women were considered carriers, but this idea was revised and today it is known that female patients can present severe manifestations of the disease. Therefore, since also heterozygous women can develop an irreversible organ damage, a careful clinical follow-up should be carried out, even if the values of enzyme activity are in the normal range.

The availability of a recombinant enzyme replacement therapy (ERT) has an important impact on clinical management of affected patients, improving the prognosis and their quality of life. The data reported in literature show the importance of a specific therapeutic intervention made as early as possible, before the organ involvement becomes irreversible. ${ }^{10}$ 
However, ERT alone does not appear to resolve or arrest some manifestations of Fabry disease and it has been recommended the use of additive therapies in FD patients, such as the paricalcitol that is able to reduce proteinuria in the presence of ERT and anti-RAS therapy. ${ }^{11}$

\section{Cardiac involvement in Fabry disease}

Cardiac involvement is frequent in Fabry disease, both in males and in females; patients often show a left ventricular hypertrophy that mimics sarcomeric hypertrophic cardiomyopathy. ${ }^{12}$ Arrhythmias, valvular alterations and conduction defects are also common in FD and they generally appear in the fourth decade of life with a progressive onset.

A study conducted on 1448 patients from the International Fabry Registry showed that left ventricular hypertrophy is present in $14 \%$ of men and $20 \%$ of women, that manifested also other signs and symptoms that are typical of FD. ${ }^{13}$ Moreover, literature reports that 2-3\% of patients with idiopathic left ventricular hypertrophy are affected by Fabry disease,,${ }^{14}$ as well as $0.5 \%$ of patients with a previous diagnosis of hypertrophic cardiomyopathy. ${ }^{15}$

Regarding the role of cardiologists in the management of Fabry patients, they are involved in many aspects: the differential diagnosis with other cardiomyopathies, the early diagnosis of hearth damage in subjects with mutations in the $G L A$ gene, the progression of cardiac damage, and the decisions concerning the beginning and the follow-up of the enzyme replacement therapy.

\section{A disease often seen but rarely diagnosed}

Fabry disease is considered a rare pathology but actually, according to the recent data in literature, it would be more correct to consider this syndrome like an uncommon disorder that is not well known. To date, the diagnosis is still difficult because of the features of the disease: its clinical manifestations overlap with the ones of other pathologies and, for this reason, there is a wide possibility of differential diagnoses made by different medical specialists. ${ }^{16}$ The difficulties in diagnosing this pathology are shown by retrospective studies that highlighted a significant diagnostic delay in about $40 \%$ of male and $70 \%$ of female patients. In particular, the average time elapsing between the beginning of first signs and symptoms and the correct diagnosis is 13 years for male patients and 17 years for female patients.
Moreover, the diagnostic error is a concrete risk and it determines an underestimation of the real number of affected subjects. These data confirm the considerations present in Hoffmann and Mayatepek's article, in which they wrote that Fabry disease is a disorder often seen but rarely diagnosed. ${ }^{17}$ Therefore screening programs in high-risk populations (composed of patients, primarily males, with end-stage renal disease, left ventricular hypertrophy, cryptogenic strokes) should be seriously taken into account; e.g., a European study revealed that the prevalence of Fabry disease is $0.264 \%$ among male dialysis patients. ${ }^{18}$

\section{Our experience}

Since 2005 our research group, operating at the Institute of Biomedicine and Molecular Immunology of the Italian National Research Council (IBIM-CNR) of Palermo, is devoted to the study of Fabry disease. In these years, we studied about 8500 subjects with a symptomatology attributable to FD, coming from many Italian clinics, and 3000 healthy controls. In our case-finding study, exonic mutations in the GLA gene were found in 190 of the analyzed subjects, the $27 \%$ of which came from Divisions of Cardiology (Figure 1). The majority of these mutations are already reported in databases as responsible for Fabry disease, while others were described by us for the first time in the world and, considering the genetic, biochemical and clinical data, they were associated to the disease. ${ }^{19-22}$ In particular, we described twelve novel mutations: three are nonsense mutations that introduce a stop codon in the messenger RNA and consequently produce a truncated protein; five are missense mutations that replace an amino acid with another one in the $\alpha$-GAL A; three are insertions or deletions that cause a frameshift and a premature termination of the translation; one is a substitution of a nucleotide with another one in the non-coding region of the

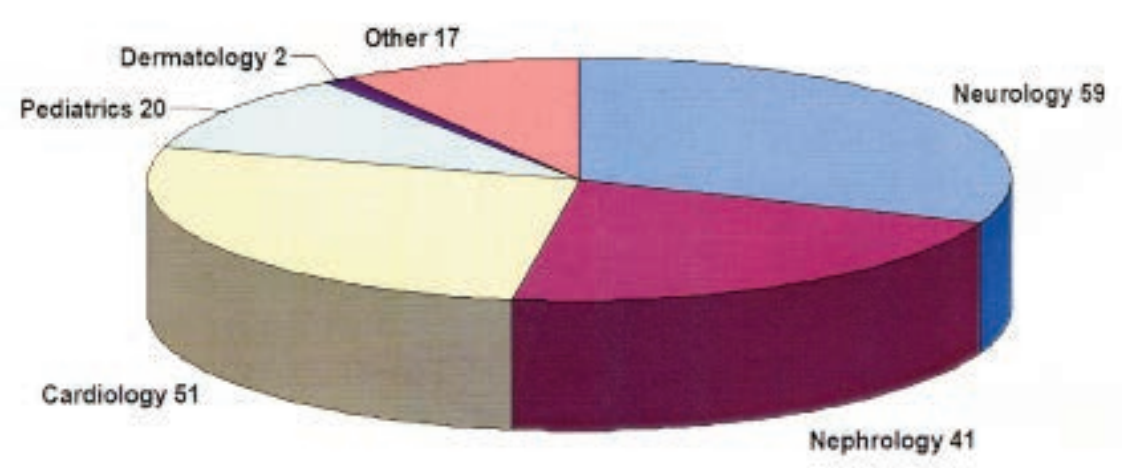

Figure 1. Distribution of patients with mutations in the GLA gene.
$G L A$ gene in a sequence involved in the regulation of gene expression. It is noteworthy that Fabry disease was promptly hypothesized only in a small percentage of affected patients that we studied, in whom the genetic investigation confirmed the disease, while the majority of them was previously misdiagnosed. The exonic mutations in the $G L A$ gene identified by us can be classified in three different groups: mutations associated to classic phenotype, mutations that cause atypical variants, and mutations not responsible for Fabry disease, according to the literature.

Fabry disease is suspected on the basis of clinical and anamnestic-familial data, and it is confirmed by measuring $\alpha$-galactosidase $\mathrm{A}$ activity, that could be null or deficient, and genetic analysis, in order to find causative mutations in the GLA gene. Moreover, a diagnostic support could be given by the determination of the enzyme substrates (Gb3 and lysoGb3) that are accumulated in the lysosomes of cells of different organs and tissues. However, we are conscious that Fabry disease could be a pathology more complex than previously considered and probably the diagnostic tests currently in use could be not always sufficient to confirm the clinical diagnosis. The complexity of the disease is also shown by the lack of correspondence, in some cases, between enzyme activity and clinical manifestations in subjects with mutations in the $G L A$ gene. Indeed, in our experience we reported many cases of aged male relatives of probands that, even if they had causative mutations in the GLA gene and no enzyme activity, did not present a severe symptomatology. Vice versa, mutations in the GLA gene responsible for Fabry phenotype were not found in the majority of the probands that we studied, even if they presented clinical manifestations attributable to the disease. In both groups of subjects, it is not clear if these individuals can be considered affected by Fabry disease. Probably, like it was reported in many articles, other molecules could be also involved in the onset of the disease, such as cofactors essential for the functionality of the $\alpha$-galac- 
tosidase A (Saposin), ${ }^{23}$ receptors for the enzyme transport to the lysosomes (mannose 6 -phosphate receptor and Sortilin), ${ }^{24}$ proteins that stabilize the $\alpha$-GAL A structure (Chaperones). ${ }^{25}$ These molecules should be considered in the study of Fabry disease. Moreover, other genes related to systemic manifestations could be studied: mutations in these genes could have a synergistic effect with the alterations in the systems involved in Fabry disease or, at least, in the symptomatology of patients, that are not necessarily Fabry.

\section{Enzyme activity}

In our laboratories the dried blood filter paper test, a method commonly used for the determination of $\alpha$-galactosidase A activity in blood, was further improved and optimized. Our data showed that in all the male Fabry patients with $G L A$ gene mutations the $\alpha$-galactosidase A activity is lower than normal value. The same correspondence was not observed in female patients because in the different cells of the organism, due to the lyonization, the enzyme activity is extremely variable from normal to pathological values. ${ }^{26}$ A retrospective study conducted by our research group showed that more than $57 \%$ of Fabry female patients had normal value of the enzyme activity. Therefore, the genetic analysis in women is the most reliable assay to confirm the clinical diagnosis of Fabry disease.

Regarding a possible relation between level of enzyme activity and severity of Fabry disease in women, it is necessary to underline that, generally, patients with null $\alpha$-GAL A activity show severe clinical manifestations; however, this does not mean that female subjects with a normal enzyme activity have no symptoms. Indeed, due to the lyonization, in some organs there could be a prevalence of cells in which the $\mathrm{X}$ chromosome with mutated GLA gene is active, thus causing organ damage.

\section{Conclusions}

The classic form of Fabry disease manifest itself in the childhood with peripheral neurological symptomatology and, with the age, it progressively involves kidney, hearth and central nervous system. This progression can have various forms and differences in the clinical manifestations: age of onset, different sign and symptoms and their severity. In some cases, the disease manifests only a mild painful symptomatology and with a minimal organ involvement: atypical variants.

Overall, patients that present clinical mani- festations referable to Fabry disease can be classified in two groups: i) subjects with causative mutations in the gene that encodes the $\alpha$-galactosidase A (GLA gene); ii) subjects with a symptomatology referable to Fabry disease but without mutations in the coding regions of the $G L A$ gene.

In the first group, the identification of a causative mutation in the coding regions of the GLA gene, together with null enzyme activity and the presence of glycosphingolipid accumulation, confirms the clinical diagnosis made by the clinician. On the other hand, in the second group, consisting of about $98 \%$ of subjects that came to our attention, it is necessary to use innovative diagnostic tools. However, it is unlikely that all these cases are Fabry patients and, realistically, at least the half of Fabry clinical diagnosis could be avoided if specific diagnostic algorithms were available for clinicians. These considerations led us to initiate some research projects that aim at identifying new specific molecular markers of Fabry disease, in order to give an important tool to clinicians for differential diagnosis. One of these projects consists in the study of expression profiles of circulating microRNAs, small molecules involved in the regulation of gene expression that are used as important and innovative diagnostic biomarkers in many pathologies. Other projects currently underway consist in proteomics and next generation sequencing studies.

In spite of the risk of an excess of diagnostic hypotheses, Fabry disease should be considered in all the patients with atypical clinical course, uncertain diagnosis, or clinical picture with systemic involvement that is not clear, in particular when a patient also shows painful crises not justified, paresthesias, heat/cold intolerance, hypohidrosis, angiokeratomas, abdominal pain and cornea verticillata. Furthermore, classic or atypical forms of the pathology can be suspected in adult subjects with cryptogenic stroke, hypertrophic cardiomyopathy or kidney damage. Also the study of clinical familial history, considering the Xlinked transmission of the disease, can help the clinician. A reliable and prompt diagnosis in Fabry patients is essential to initiate early the enzyme replacement therapy, which is able to slow down or to arrest the progression of the disease, improving the quality of life. To date, the clinical diagnosis of Fabry disease is confirmed by genetic and biochemical analyses. The determination of Gb3 and lyso-Gb3 storage can also confirm the clinical diagnosis, but it is desirable the advent of other specific markers that could take account of other factors potentially involved.

In conclusion, in the last years, the knowledge of the disease is considerably increased and for this reason we are conscious that it could be more complex than previously consid- ered, and maybe many subjects with a symptomatology referable to Fabry disease could be affected by a different pathology.

\section{References}

1. Brady R0, Gal AE, Bradley RM, et al. Enzymatic defect in Fabry's disease. Ceramidetrihexosidase deficiency. N Engl J Med 1967;276:1163-7.

2. Desnick RJ, Ioannou YA, Eng CM. Alphagalactosidase A deficiency: Fabry disease. In: Scriver CR, Beaudet AL, Sly WS, Valle $\mathrm{D}$, eds. The metabolic and molecular basis of inherited disease. 8th ed. New York, NY: McGraw-Hill; 2001. pp 3733-74.

3. Spada M, Pagliardini S, Yasuda M, et al. High incidence of later-onset fabry disease revealed by newborn screening. Am J Hum Genet 2006;79:31-40.

4. Hwu WL, Chien YH, Lee NC, et al. Newborn screening for Fabry disease in Taiwan reveals a high incidence of the later-onset GLA mutation c. $936+919$ G $>$ A (IVS4+9 19G>A). Hum Mutat 2009;30: 1397-405.

5. Kornreich R, Bishop DF, Desnick RJ. The gene encoding alpha-galactosidase $\mathrm{A}$ and gene rearrangements causing Fabry disease. Trans Assoc Am Physicians 1989;102: 30-43.

6. Germain DP. Fabry disease. Orphanet J Rare Dis 2010;5:30.

7. Pisani A, Visciano B, Imbriaco M, et al. The kidney in Fabry's disease. Clin Genet 2014;86:301-9.

8. Faggiano A, Pisani A, Milone F, et al. Endocrine dysfunction in patients with Fabry disease. J Clin Endocrinol Metab 2006;91:4319-25.

9. Maione L, Tortora F, Modica R, et al. Pituitary function and morphology in Fabry disease. Endocrine 2015;50:483-8.

10. Pisani A, Visciano B, Roux GD, et al. Enzyme replacement therapy in patients with Fabry disease: state of the art and review of the literature. Mol Genet Metab 2012;107:267-75.

11. Pisani A, Sabbatini M, Duro G, et al. Antiproteinuric effect of add-on paricalcitol in Fabry disease patients: a prospective observational study. Nephrol Dial Transplant 2015;30:661-6.

12. Gambarin FI, Disabella E, Narula J, et al. When should cardiologists suspect Anderson-Fabry disease? Am J Cardiol 2010;106:1492-9.

13. Pinderski LJ, Strotmann J. Congestive heart failure in Fabry cardiomyopathy: natural history experience in an international cohort of 1,448 patients. J Heart Lung Transpl 2006;25:S70.

14. Sachdev B, Takenaka T, Teraguchi H, et al. 
Prevalence of Anderson-Fabry disease in male patients with late onset hypertrophic cardiomyopathy. Circulation 2002;105: 1407-11.

15. Elliott P, Baker R, Pasquale F, et al. Prevalence of Anderson-Fabry disease in patients with hypertrophic cardiomyopathy: the European Anderson-Fabry Disease survey. Heart 2011;97:1957-60.

16. Marchesoni CL, Roa N, Pardal AM, et al. Misdiagnosis in Fabry disease. J Pediatr 2010;156:828-31.

17. Hoffmann B, Mayatepek E. Fabry diseaseoften seen, rarely diagnosed. Dtsch Arztebl Int 2009;106:440-7.

18. Kotanko P, Kramar R, Devrnja D, et al. Results of a nationwide screening for Anderson-Fabry disease among dialysis patients. J Am Soc Nephrol 2004;15:13239.

19. Colomba P, Nucera A, Zizzo C, et al.
Identification of a novel mutation in the alpha-galactosidase A gene in patients with Fabry disease. Clin Biochem 2012;45:839-41.

20. Tuttolomondo A, Duro G, Miceli S, et al. Novel alpha-galactosidase A mutation in a female with recurrent strokes. Clin Biochem 2012;45:1525-30.

21. Duro G, Musumeci MB, Colomba P, et al. Novel $\alpha$-galactosidase $A$ mutation in patients with severe cardiac manifestations of Fabry disease. Gene 2014;535:3659.

22. Zizzo C, Monte I, Pisani A, et al. Molecular and clinical studies in five index cases with novel mutations in the GLA gene. Gene 2015 [In press].

23. Kase R, Bierfreund U, Klein A, et al. Only sphingolipid activator protein B (SAP-B or saposin B) stimulates the degradation of globotriaosylceramide by recombinant human lysosomal alpha-galactosidase in a detergent-free liposomal system. FEBS Lett 1996;393:74-6.

24. Prabakaran T, Nielsen R, Satchell SC, et al. Mannose 6-phosphate receptor and sortilin mediated endocytosis of $\alpha$-galactosidase A in kidney endothelial cells. PLoS One 2012;7:e39975.

25. Yue P, Li Z, Moult J. Loss of protein structure stability as a major causative factor in monogenic disease. J Mol Biol 2005;353: 459-73.

26. Dobrovolny R, Dvorakova L, Ledvinova J, et al. Relationship between X-inactivation and clinical involvement in Fabry heterozygotes. Eleven novel mutations in the alpha-galactosidase A gene in the Czech and Slovak population. J Mol Med 2005;83: 647-54. 